\title{
The Information Service Technology of Electronic Information System
}

\author{
Xianyu Li, Hua Wang, Jianqun Zheng, Zhiyuan Chen, Qiang Chen \\ Beijing Shi QingHe DaLou Zi Jiu, Beijing, 100085, China \\ email: 919wh_cn@sina.com
}

Keywords: Information Service Technology; Service Architecture; Electronic Information System

\begin{abstract}
Based upon the synopsis of electronic information system and information services, this paper puts forward the attributes and the logic structure of information service, sets forth the service architecture of electronic information system, and presents a series of measures to improve information service system, such as business information flow optimization, metadata data sharing standard establishment, common basic service development and information service environment security. This will benefit the continual improvement of information service system and the further development of electronic information system.
\end{abstract}

\section{Introduction}

Electronic information system is a distributed man-machine system that effectively organizes related resources, conducts information processing and actualizes business activities. Service-oriented architecture (SOA) can translate function units of different applications into information services among that interfaces and protocols are favorably defined, and the information services are the integration basis of electronic information system [1][2].

Along with the continuous progress of information technology, electronic information system need further satisfy the requirements of dynamic function evolvement and mission realization, and meantime the practices and development of information service system can provide available technical approaches and overall project for it. Thus the investigation of the information service framework and related measures of electronic information system is no doubt meaningful.

\section{Information Service Resume}

Information service attributes generally include the function attribute of kernel metadata and message format, and the non-function attribute of service state, service quality and user-defined [3].

(1) Kernel metadata is the descriptive information that pictures the service characteristics of status, content and copyright.

(2) Message format is the communication protocol that serves when service applicant transfer related services.

(3) Service state is to describe whether present service is available.

(4) Service quality is the capability of information service to meet user requirements or complete stated task, and includes the defined static parameters of robustness, scalability and security, and the supervised dynamic indexes of accuracy, delay and reliability.

(5) User-defined is for the purpose of extending the description of information service.

And information service is the encapsulation entity of system function unit, which comprise three logic layers such as description layer, entity layer and interface layer, as is shown in Figure 1.

(1) Interface layer specifies the outer interfaces of information service that comprise communication protocol, message format and data type, and actualizes the separation between service interfaces and functions.

(2) Entity layer includes the function realization and resources encapsulation, and provides the related service applications.

(3) Description layer provides the description information needed for the service discovery and transfer. 


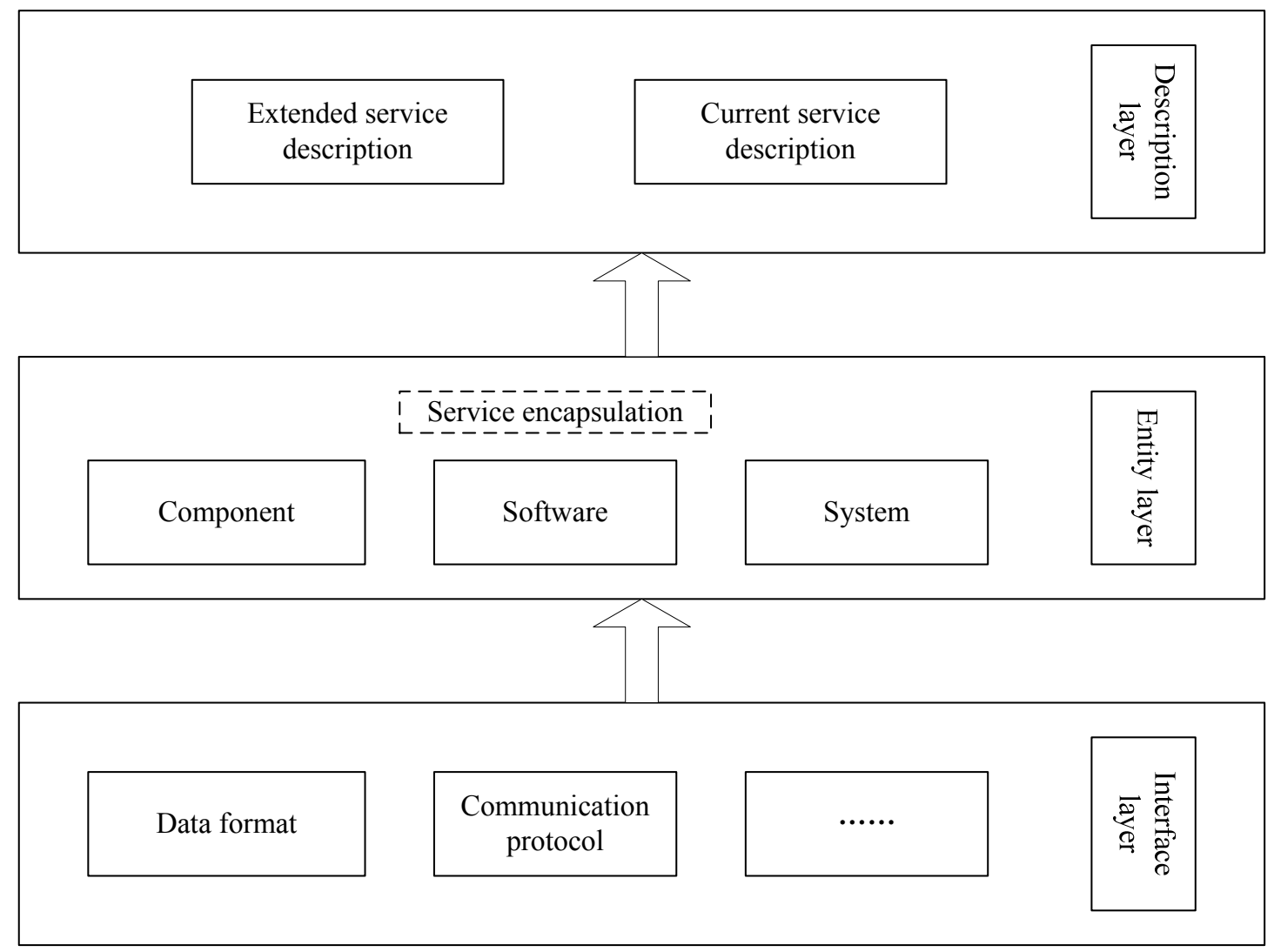

Fig.1. The logic structure of information service

\section{System Service Architecture}

Information service system contains various integrative management and business services that support the related infrastructure based on service oriented technology [4].

Based on the information service system, the service architecture of electronic information system usually includes application layer, service management layer, information service layer and physical resources layer, as is shown in Figure 2.

(1) Application layer comprises general business and special mission modes with respect to particular business requirements, and combines the related independent and distributed information services and constitute the whole system.

(2) Service management layer is to ensure service deploying, operation, integration, monitoring and regroupment and performance analysis, and accordingly it can separate construction process from special bottom services and focus on system flow and business logics.

(3) Business service layer mainly deals with the whole process of message request and message receipt via data service interfaces and integration data visit, and consists of various business services, service description, encapsulation, monitoring and etc.

(4) Physical resources layer provides a basis for the implementation of information services, and comprises various supportive infrastructures, such as server, computer, network, storage equipment and so on.

Service oriented electronic information system is substantially based on various information services distributed in a network in light of mission requirements, and integrated into a mutual operational system by means of service combination and transfer, alternation and cooperation. 


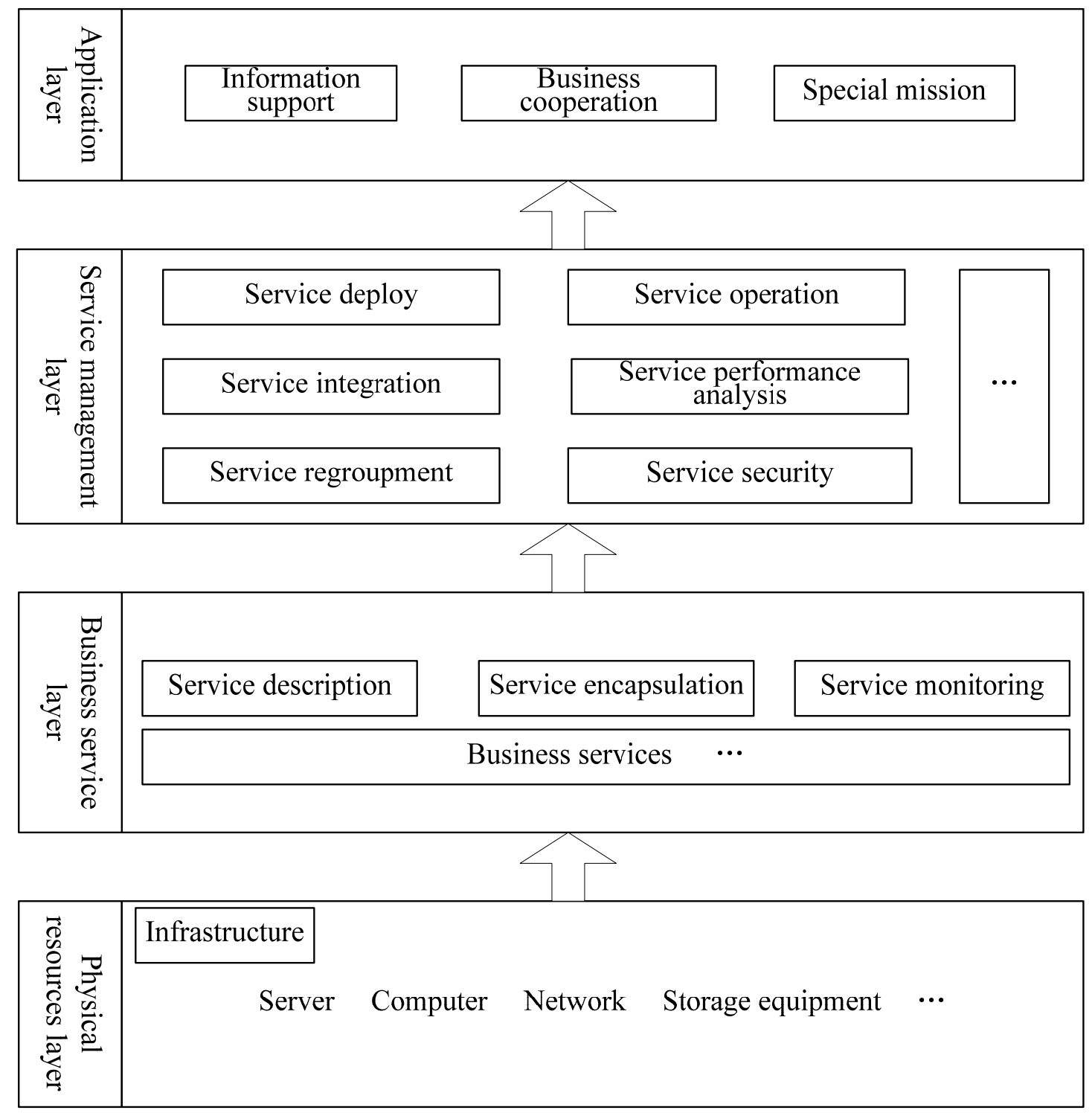

Fig.2. The service architecture of electronic information system

The distributed functions and resources are depicted and encapsulated into independent information services with unitive interface standard; the alternation and communication among services are designed via service bus topology to advance system data and function sharing; and the information services are recombined and aggregated according to certain operational business flow, and eventually transferred, alternated and collaborated so as to complete the desired smart and dynamic system.

\section{Measures}

According to the top scheme and related standards, we need take a series of measures to smoothly accelerate information service system and continue to improve electronic information system [5][6], and the related measures are mainly as follows.

(1) Business information flow optimization.

We should optimize business information flow with respect to the related processes of information collecting, transferring, processing, feedback and control according to new situation and mission, utilize various technology means of parallel programming mode, distributed resource management, and carry out system architecture design, quality elevation of software design and realization, and improvement of program arithmetic efficiency so as to arrive at the effective decision and agile operation.

(2) Metadata data sharing standard establishment. 
In light of the top scheme, we should compile metadata data sharing standards, so that we can process data maintainment in every business application field, and reduce service complexity in the whole field. And by means of field data issue, sharing space creation and metadata catalog, system private data will be changed into field sharing and entireness sharing step by step, so that users can fast detect and make use of system data at any moment and everywhere.

(3) Common basic service development.

Common basic services are the important sustainment of system operation. It can provide a set of common information services and capabilities, and insure data visibility, access and apprehension. We should expedite the development of common basic services, such as service registration, catalog, detection, cooperation and agency, build up basic system services and capabilities, and provide more users with effective information services via distributed service configuration.

(4) Information service environment security.

The security frame should sustain the mutual operation among various business fields on the basis of a serious of new standards, and provide practical security protection for system information services. Based on the present security infrastructure, we need establish a set of common security services that encapsulate enterprise level security function and have no relation with platform and technology, so as to solve the difficulties such as resource attributes, safe exchange among fields and access control, and ensure users the unitive cooperation and information sharing in security.

\section{Conclusions}

In the light of the overall scheme and practical demands of electronic information system, we should fully promote the application of information service technologies to the system integration and development. In addition, we should optimize business information flow, implement metadata data sharing standards, advance common basic service development, and enhance information service environment security. It is the complete scheme, advisable technologies and appropriate standards of system information services that would fully ensure system collective capabilities.

\section{Acknowledgement}

We are grateful to the experts and secretaries for their guidance, understanding and patience; they are a constant source of support and encouragement.

\section{References}

[1] Chuanqing Cai, Guangqi Huang. Unified Method for Describing Military Information Services [J]. Command Information System and Technology, 2011(2) 53-56.

[2] Wei Jung, Zifeng Cui. Net-Centric Environment of Information Service and Its Development in Military Field [J]. Command Information System and Technology, 2011(4) 10-16.

[3] Zhen Shu and so on. Research on Concept of Military Information Service and its Application [J]. Fire Control \& Cornrnand Control, 2010 (9) 53-57.

[4] Zhang P Y, Huang B, Sun Y M. Petri-net-based description and verification of Web services composition model [J]. Journal of System Simulation, 2007 ( 2) 2872-2876.

[5] Defense Information Systems Agency. Net-Centric Enterprise Services Overview [M]. Department of Defense, 2004.

[6] Liu Y T, Ngu Anne H H, Zeng L Z. QoS computation and policing in dynamic web service selection [C]. Proceedings of the 13th International World Wide Web Conference (WWW 2004). ACM Press, 2004: 798-805. 